\title{
Dapagliflozin attenuates renal gluconeogenic enzyme expression in obese rats
}

\author{
Myat Theingi Swe 1,2, Laongdao Thongnak'1, Krit Jaikumkao ${ }^{3}$, Anchalee Pongchaidecha', Varanuj Chatsudthipong ${ }^{4}$ \\ and Anusorn Lungkaphin 1
}

1Department of Physiology, Faculty of Medicine, Chiang Mai University, Chiang Mai, Thailand 2Department of Physiology, University of Medicine 2, Yangon, Myanmar

${ }^{3}$ Department of Radiologic Technology, Faculty of Associated Medical Sciences, Chiang Mai University, Chiang Mai, Thailand

4Research Center of Transport Protein for Medical Innovation, Faculty of Science, Mahidol University, Bangkok, Thailand

Correspondence should be addressed to A Lungkaphin: onanusorn@yahoo.com

\begin{abstract}
The kidneys release glucose into the systemic circulation through glucose reabsorption and renal gluconeogenesis. Currently, the significance of renal glucose release in pathological conditions has become a subject of interest. We examined the effect of sodium-dependent glucose cotransporter 2 inhibitor (SGLT2i) on renal gluconeogenic enzyme expression in obese rats. Male Wistar rats (180-200 g) were fed either a normal $\operatorname{diet}(\mathrm{ND}, n=6)$ or a high-fat diet. At 16 weeks, after confirming the degree of glucose intolerance, high-fat diet-fed rats were randomly subdivided into three groups ( $n=6 /$ group): untreated group (HF), treated with dapagliflozin $1 \mathrm{mg} / \mathrm{kg} /$ day (HFSG) and treated with metformin $30 \mathrm{mg} / \mathrm{kg} /$ day (HFM). The treatment was continued for 4 weeks. We observed that dapagliflozin or metformin mitigated the enhanced expression of renal gluconeogenic enzymes, PEPCK, G6Pase and FBPase, as well as improved glucose tolerance and renal function in obese rats. Dapagliflozin downregulated the elevated expression of gluconeogenic transcription factors p-GSK3 $\beta$, p-CREB and coactivator $\mathrm{PGC} 1 \alpha$ in the renal cortical tissue. Metformin reduced the expression levels of renal cortical FOXO1 and CREB. Furthermore, reduced renal insulin signaling was improved and renal oxidative stress was attenuated by either dapagliflozin or metformin treatment in obese rats. We concluded that glucose tolerance was improved by dapagliflozin in obese prediabetic rats by suppressing renal glucose release from not only glucose reabsorption but also renal gluconeogenesis through improving renal cortical insulin signaling and oxidative stress. The efficacy of dapagliflozin in improving renal insulin signaling, oxidative stress and renal function was greater than that of metformin.
\end{abstract}

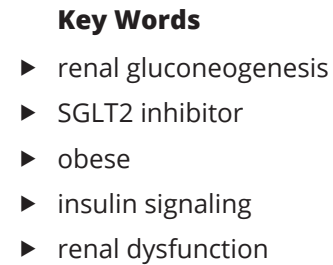

Journal of Endocrinology (2020) 245, 193-205

\section{Introduction}

The role of kidneys in glucose homeostasis under physiological conditions has long been acknowledged. The kidneys are involved in glucose metabolism via three distinct processes: glycolysis for renal glucose uptake, gluconeogenesis, and glucose reabsorption for renal glucose release (Mitrakou 2011). The amount of glucose released from the kidneys into the systemic circulation is determined by the balance between these processes. Currently, the significance of renal glucose release in pathological conditions has become the subject of interest.

The renal medullary portion takes up glucose and relies exclusively on glucose as a metabolic fuel source. 
In contrast, the renal cortical portion synthesizes and releases new glucose into the circulation via renal gluconeogenesis (Gerich 2010). During the postabsorptive period, the amount of glucose released from renal gluconeogenesis is approximately $20-25 \%$ of the total endogenous glucose production (EGP) (Gerich et al. 2001). In the postprandial period, the renal gluconeogenesis increases by two-fold, accounting for nearly $60 \%$ of EGP, while the hepatic glucose release is suppressed to enable the efficient repletion of the liver glycogen stores after a meal (Meyer et al. 2002). Therefore, the contribution of renal gluconeogenesis to plasma glucose levels should not be neglected.

The rate-limiting enzymes for gluconeogenesis are phosphoenolpyruvate carboxykinase (PEPCK), glucose6-phosphatase (G6Pase) and fructose-1,6-bisphosphatase (FBPase). The activities of these gluconeogenic enzymes are mostly regulated by the rate of gene transcription and the level of protein expression (Mithieux et al. 1996, Quinn \& Yeagley 2005). The regulation of gluconeogenesis occurs mostly through the metabolic hormones and the alterations in the availability of gluconeogenic substrates (Meyer et al. 1998, DeFronzo et al. 2012). Insulin is the principal negative regulator of renal gluconeogenesis and a variety of signaling pathways modulated by insulin converge at the transcriptional regulation of gluconeogenic genes (Swe et al. 2019a).

Reduction in plasma insulin levels during the fasting period exerts a permissive effect on gluconeogenesis to maintain plasma glucose levels. However, when there is insulin deficiency or insulin resistance, abnormally elevated EGP under fasting and/or abnormal persistence of this process during the postprandial period becomes the major contributor to hyperglycemia (Rizza 2010, Kacerovsky et al. 2011). In type 2 diabetes, glucose released from renal gluconeogenesis is enhanced (Mitrakou 2011, Tojo et al. 2015) both in the fasting (Meyer et al. 1998) and postprandial periods (Meyer et al. 2004). Interestingly, obesity is pivotal to the future development of type 2 diabetes, while insulin resistance is one of the major links between obesity and type 2 diabetes (Khaodhiar et al. 2009), and obesity is considered a global health disaster nowadays. Therefore, exploring the renal gluconeogenesis in an obese condition where insulin signaling is compromised is interesting.

The sodium-dependent glucose cotransporters, SGLT1 and 2 , in the renal proximal tubule reabsorb nearly all filtered glucose. SGLT2 is responsible for approximately $90 \%$ of the glucose reabsorption in the kidneys (Mather \& Pollock 2011), and selective pharmacological inhibition of SGLT2 in type 2 diabetes lowers blood glucose by inducing glycosuria. Over the past few years, it has become increasingly clear that SGLT2i (SGLT2 inhibitors) possess pleiotropic actions beyond their glycosuric action. The SGLT2 inhibitor dapagliflozin reportedly improves diabetic nephropathy through improving albuminuria, oxidative stress, inflammation and fibrosis in clinical and preclinical studies (Terami et al. 2014, Shin et al. 2016, Yoshihara et al. 2018).

Despite the growing evidence of SGLT2i pleiotropic actions, their effects on renal gluconeogenesis in a prediabetic obese condition have not been well examined. The aim of the current study was to investigate the effect of dapagliflozin on the expression levels of renal cortical gluconeogenic enzymes in high-fat diet-fed obese rats. In addition, the possible underlying molecular mechanisms by which dapagliflozin affected renal gluconeogenesis in obesity were explored.

\section{Materials and methods}

\section{Animals}

The study protocol was reviewed and permitted by the Laboratory Animal Care and Use Committees, Faculty of Medicine, Chiang Mai University, Thailand. All animal experiments were conducted at the Animal Facility, Faculty of Medicine, Chiang Mai University, Thailand, under permit number 32/2561.

Male Wistar rats at 6-8 weeks of age weighing 180-200 g (Nomura Siam International, Bangkok, Thailand) were maintained on a standard rat chow $(19.77 \%$ of energy from fat) (ND, $n=6)$ or high-fat diet (59.28\% of energy from fat) (n=18) ad libitum and had free access to food and tap water. Before this, all rats were given standard rat chow for 1 week as an acclimatization period. A high-fat diet was fed for 16 weeks to induce an obese prediabetic condition. Diet composition was adopted from a previous study (Wanchai et al. 2018a). The rats were housed in cages in a light- and temperature-controlled environment (12 h light: $12 \mathrm{~h}$ darkness cycles and $25 \pm 1^{\circ} \mathrm{C}$ ).

At 16 weeks, an oral glucose tolerance test (OGTT) was performed to assess glucose tolerance. After confirming the level of glucose intolerance, the obese prediabetic rats were randomly divided into three groups ( $n=6 /$ group): HF (untreated high-fat diet-fed rats), HFSG (high-fat dietfed rats treated with dapagliflozin $1 \mathrm{mg} / \mathrm{kg} /$ day) and HFM (high-fat diet-fed rats treated with metformin $30 \mathrm{mg} / \mathrm{kg} /$ day). Dapagliflozin (AstraZeneca (Thailand) Limited) and metformin (Glucophage, Bangkok, Thailand) were freshly dissolved with normal saline and administered by oral 
gavage for 4 weeks, while untreated rats were given normal saline only. The dose of each drug was chosen based on the previous study (Jaikumkao et al. 2018). Energy intake and body weight were recorded daily throughout the experiment.

\section{Blood, urine and tissue collection}

At 20 weeks, the 24-h urine collection was done using metabolic cages, while free access to water and food was permitted. Following a 12-h overnight fasting, the killing was done by an overdose of isoflurane inhalation which was maintained until the breathing stopped. Blood samples were collected from the inferior vena cava and stored at $-20^{\circ} \mathrm{C}$ until analysis. Killing was confirmed by harvesting organs including the vital organs. Visceral fat and kidney weights were recorded. Kidney cortical tissues were sliced and stored at $-80^{\circ} \mathrm{C}$ for subsequent Western blotting and tissue malondialdehyde (MDA) measurement.

\section{Oral glucose tolerance test and homoeostasis model assessment of insulin resistance (HOMA-IR)}

The oral glucose tolerance test was performed twice at 16 weeks and 20 weeks to assess the degree of glucose tolerance. After overnight fasting, a glucose load $(2 \mathrm{~g} / \mathrm{kg}$ body weight) was given by oral gavage. A blood sample was collected from the tail vein under brief anesthesia at 0, 30, 60 and 120 min after glucose ingestion and stored at $-20^{\circ} \mathrm{C}$ for measuring blood glucose levels. The extent of glucose tolerance was evaluated calculating the values for plasma glucose area under the curve (AUCg) using the trapezoidal formula. Insulin resistance at 20 weeks was assessed by homoeostasis model assessment for insulin resistance (HOMA-IR) which was calculated using the values of fasting plasma glucose and fasting plasma insulin by the following formula: HOMA-IR=(fasting insulin level $(\mathrm{ng} / \mathrm{mL}) \times$ fasting glucose level $(\mathrm{mg} / \mathrm{dL})) / 405$ (Roza et al. 2016).

\section{Measurement of blood and urine parameters}

The glucose levels in the blood and urine were measured using a colorimetric assay kit (Erba Diagnostics Mannheim $\mathrm{GmbH}$, Mannheim, Germany), and the plasma insulin level was measured using a rat insulin ELISA kit (Millipore). Enzymatic colorimetric method using a commercial assay kit (DiaSys Diagnostic Systems GmbH, Holzheim, Germany) was chosen to determine the serum and urine creatinine levels. The detection of urine microalbumin was performed using the commercial kit (Roche Diagnostics). The plasma reduced glutathione (GSH) level was assessed using a commercial kit (Bioassay Systems, Hayward, CA, USA) according to the manufacturer's instructions. The serum and renal cortical malondialdehyde (MDA) levels were determined using a thiobarbituric acid reactive substances (TBARS) assay kit (Cayman Chemical Company).

\section{Western blotting}

The renal cortical tissues were homogenized to extract whole-cell kidney lysate and membrane fractions. Cell fractionation was achieved by differential centrifugation at $5000 \boldsymbol{g}$ for $10 \mathrm{~min}$ (supernatant for whole-cell kidney lysate fraction) followed by $100,000 \boldsymbol{g}$ for $2 \mathrm{~h}$ (pellet for membrane fraction). The protein concentration in each sample was measured by a Bio-Rad protein assay kit (BioRad Laboratories Inc.).

Samples containing an equal amount of protein were resolved on a $10 \%$ or $12 \%$ gel (Bio-Rad Laboratories Inc.) and electroblotted to polyvinylidene fluoride (PVDF) membranes (Millipore). The membranes were subsequently incubated with 5\% non-fat dried milk in Tris-buffered saline containing $0.1 \%$ Tween 20 (TBST) or PBS containing $0.1 \%$ Tween 20 (PBST) followed by overnight incubation with primary antibodies. After that the membranes were incubated with the specific horseradish peroxidase (HRP)conjugated secondary antibody (Millipore) to visualize the antigen-antibody reaction. The luminescent signal was detected by exposure to Amersham Hyperfilm ${ }^{\mathrm{TM}}$ ECL (GE Healthcare Limited). Densities of the bands were analyzed using ImageJ software (National Institutes of Health, Bethesda, MD, USA). Then, the membranes were stripped and reprobed with an anti-GAPDH antibody for loading control.

The primary antibodies were purchased as follows: PEPCK (1:1000) from Abcam; G6Pase (1:330) from Thermo Fisher Scientific; CREB (1:200), p-CREB (1:250), PGC1 $\alpha$ (1:500), p38 (1:250), p-p38 (1:200), p-IRS1 (1:200), IRS2 (1:250), Akt (1:500), GSK3 $\beta$ (1:250), p-GSK3 $\beta$ (1:250), JNK (1:400) and GAPDH (1:2000) from Millipore; NOX4 (1:200) from Santa Cruz Biotechnology and FBPase (1:1000), FOXO1 (1:200), AMPK (1:250), p-AMPK (1:200), PI3K (1:250), p-Akt (1:500), ERK1/2 (1:1000) and p-ERK1/2 (1:200) from Cell Signaling Technology.

\section{Immunohistochemical staining}

Immunostaining was performed on paraffin-embedded renal tissue. Briefly, the paraffin blocks were sliced into sections, deparaffinized with xylene and rehydrated with 
ethanol. Antigen unmasking was achieved by incubating the slides in Tris-ethylenediaminetetraacetic acid (EDTA) buffer for $20 \mathrm{~min}$ in a water bath at $92.5^{\circ} \mathrm{C}$. For cell permeability, the slides were incubated with $0.3 \%$ Triton $\mathrm{X}$ in Tris-buffered saline (TBS) for $1 \mathrm{~h}$. Endogenous peroxidases and biotin were then blocked by $0.3 \%$ hydrogen peroxide and $5 \%$ fetal bovine serum, respectively. Primary antibody, G6Pase (1:150) (Thermo Fisher Scientific) or p-CREB (1:150) (Millipore), was applied overnight at $4^{\circ} \mathrm{C}$. Then, the slides were incubated with secondary antibody biotinylated goat anti-rabbit IgG (Millipore) for $1 \mathrm{~h}$ at room temperature. After the slides were incubated with horseradish peroxidase (HRP) reagent, color development was achieved by applying the diaminobenzidine tetrahydrochloride (DAB) solution. Then, the slides were counterstained with hematoxylin (HiMedia Laboratories Pvt. Ltd., Mumbai, India) and examined under an Olympus light microscope (Olympus America Inc.) (magnification 20x) by an observer blinded to the animal treatment group. For scoring, the frequency of staining was determined using the following scale: $0=$ no or hardly any cells positive, $1=$ small fraction of cells positive, $2=$ approximately half of the cells positive, $3=$ more than half of the cells positive and $4=$ all or the majority of cells positive ( $n=5$ fields/group) (Schulkens et al. 2014).

\section{Statistical analysis}

Statistical analysis was done using SPSS Statistics for Windows, v22.0 (SPSS Inc.). Data were shown as mean \pm s.E.M. The plasma AUCg level at 16 weeks was compared between ND and high-fat diet-fed rats using an independent sample $t$-test. Otherwise, the significance of the differences was estimated using ANOVA with Fisher's least significant difference (LSD) post hoc test. A $P$ value of $<0.05$ was regarded as indicating statistical significance.

\section{Results}

After 16 weeks of high-fat diet feeding, the plasma glucose AUCg in OGTT was significantly higher in highfat diet-fed rats than ND rats $(17.61 \pm 1.98$ vs $22.76 \pm 1.73$, $\left.\mathrm{mg} / \mathrm{dL} \times \min \times 10^{3}, \quad P<0.05\right)$, which can be considered glucose intolerance in these obese rats.

\section{Dapagliflozin attenuated body weight and visceral fat accumulation in obese rats}

As presented in Table 1, energy intake, body weight and visceral fat weight were significantly increased in untreated high-fat diet-fed rats in comparison with normal diet-fed rats $(P<0.05)$. Despite the comparable energy intake, dapagliflozin- or metformin-treated obese rats showed a reduction in body weight and visceral fat weight compared to untreated obese rats $(P<0.05)$. We observed that dapagliflozin had more efficacy in reducing visceral fat accumulation than metformin $(P<0.05)$.

\section{Dapagliflozin mitigated obesity-related renal dysfunction}

Although the kidney weights were similar among the experimental groups, obese untreated rats displayed a decrease in kidney weight/body weight ratio when compared to ND and HFSG group $(P<0.05)$ (Table 1$)$. HFSG group showed a significantly higher volume of 24-h urine excretion $(P<0.05)$ (Table 1$)$ which was parallel with an

Table 1 Effect of dapagliflozin on metabolic parameters and renal function in male Wistar rats.

\begin{tabular}{l}
\hline Variable \\
\hline Energy intake $(\mathrm{kcal} /$ day $)$ \\
Body weight $(\mathrm{g})$ \\
Visceral fat weight $(\mathrm{g})$ \\
Kidney weight $(\mathrm{g})$ \\
Kidney weight/100 g body \\
weight $(\mathrm{g})$ \\
24-h urine volume $(\mathrm{mL} / 24 \mathrm{~h})$ \\
Serum creatinine $(\mathrm{mg} / \mathrm{dL})$ \\
Creatinine clearance $(\mathrm{mL} / \mathrm{min})$ \\
Microalbuminuria $(\mathrm{mg} / \mathrm{g})$ \\
Plasma glucose $\mathrm{AUCg}$ \\
(mg/dL $\times$ min $\left.\times 10^{3}\right)$
\end{tabular}

\begin{tabular}{c}
\hline $\mathbf{N D}($ mean \pm S.E.M. $)$ \\
\hline $93.76 \pm 1.58$ \\
$538.33 \pm 17.01$ \\
$17.77 \pm 1.07$ \\
$1.18 \pm 0.04$ \\
$0.22 \pm 0.01$
\end{tabular}

\begin{tabular}{c}
\hline $\mathbf{H F}($ mean \pm S.E.M.) \\
\hline $152.73 \pm 0.49 \mathrm{a}$ \\
$751.67 \pm 51.47^{\mathrm{a}}$ \\
$62.8 \pm 6.06^{\mathrm{a}}$ \\
$1.12 \pm 0.04$ \\
$0.15 \pm 0.01^{\mathrm{a}}$
\end{tabular}

\begin{tabular}{c}
\hline HFSG $($ mean \pm S.E.M. $)$ \\
\hline $149.75 \pm 2.57 \mathrm{a}$ \\
$627.5 \pm 26.51^{\mathrm{b}}$ \\
$34.49 \pm 2.05^{\mathrm{a}, \mathrm{b}}$ \\
$1.2 \pm 0.04$ \\
$0.19 \pm 0.01^{\mathrm{b}}$
\end{tabular}

HFM $($ mean \pm S.E.M. $)$
$149.69 \pm 3.69 \mathrm{a}$
$615 \pm 48.01 \mathrm{~b}$
$49.28 \pm 5.22^{\mathrm{a}, \mathrm{b}, \mathrm{c}}$
$1.08 \pm 0.04$
$0.16 \pm 0.01^{\mathrm{a}}$

$15.67 \pm 0.42$

$0.29 \pm 0.03$

$2.71 \pm 0.29$

$10.45 \pm 0.88$

$15.68 \pm 0.82$
$24.00 \pm 2.31$

$0.46 \pm 0.03$

$5.21 \pm 0.84^{a}$

$50.59 \pm 5.26^{a}$

$22.70 \pm 2.06^{a}$
$32.00 \pm 1.93^{a, b}$

$0.36 \pm 0.02^{a, b}$

$2.86 \pm 0.08^{b}$

$34.35 \pm 1.33^{a, b}$

$16.36 \pm 0.94 b$
$20.33 \pm 1.82^{c}$

$0.41 \pm 0.02^{\mathrm{a}}$

$2.48 \pm 0.31^{b}$

$42.96 \pm 6.67 \mathrm{a}$

$19.03 \pm 0.37 b$

Male Wistar rats (180-200 g) were given a normal diet (ND) or a high-fat diet (HF) for 20 weeks. HF rats were given vehicle normal saline or 1 mg/kg/day of dapagliflozin or $30 \mathrm{mg} / \mathrm{kg} /$ day of metformin during the last 4 weeks. Data are presented as mean \pm S.E.M. Multiple comparisons were performed by one-way ANOVA followed by Fisher's least significant difference test. $n=6$ rats/group. a $P<0.05$ vs ND; $b P<0.05$ vs HF; $c P<0.05$ vs HFSG.

$\mathrm{HF}$, untreated high-fat diet rats; HFM, high-fat diet rats treated with metformin; HFSG, high-fat diet rats treated with dapagliflozin; ND, normal diet rats.

https://joe.bioscientifica.com

https://doi.org/10.1530/JOE-19-0480 (c) 2020 Society for Endocrinology Published by Bioscientifica Ltd. Printed in Great Britain 
increase in urinary glucose excretion (Fig. 1A). As shown in Table 1, HF rats had a higher serum creatinine level and creatinine clearance than ND rats $(P<0.05)$ which were significantly reduced by dapagliflozin $(P<0.05)$. Metformin also decreased the creatinine clearance in obese rats $(P<0.05)$. Urine microalbumin was significantly increased in HF, HFSG and HFM groups compared with the ND group $(P<0.05)$. However, the administration of dapagliflozin in obese rats, compared to untreated rats, significantly reduced the elevation in microalbuminuria $(P<0.05)$. The higher creatinine clearance suggests hyperfiltration in untreated obese rats, while the higher level of serum creatinine and microalbuminuria indicated an impairment of renal function. In view of these data, dapagliflozin had a greater efficacy in improving renal function than metformin.

\section{Dapagliflozin improved glucose tolerance and insulin sensitivity in obese rats}

Due to the inhibition of renal glucose reabsorption by SGLT2 cotransporter, dapagliflozin led to a marked increase in urinary glucose excretion in HFSG rats when compared with ND, HF and HFM groups $(P<0.05)$ (Fig. 1A).
Fasting plasma glucose levels in untreated obese rats did not significantly differ from the other three groups (Fig. 1B). Interestingly, HF rats had a higher level of insulin and HOMA-IR when compared with ND, HFSG and HFM groups $(P<0.05)$, displaying insulin resistance in these untreated rats (Fig. 1C and D). Although the untreated HF rats had insulin resistance, fasting plasma glucose level was not elevated probably due to compensatory hyperinsulinemia in these rats. In addition, these HF rats showed glucose intolerance by a significant elevation of AUCg of plasma glucose in OGTT in comparison with the other groups $(P<0.05)$ (Fig. $1 \mathrm{E}$ and Table 1$)$. We noticed that both dapagliflozin and metformin reduced the hyperinsulinemia and HOMA-IR as well as improved glucose tolerance in OGTT $(P<0.05)$.

\section{Obesity-related elevation in the renal gluconeogenic enzyme expression was reduced by dapagliflozin}

The expression levels of key gluconeogenic enzymes, PEPCK, G6Pase and FBPase, were simultaneously elevated in the renal cortical tissues in the high-fat diet-induced obese rat $(P<0.05)$ (Fig. 1F, G and H). These obesity-related
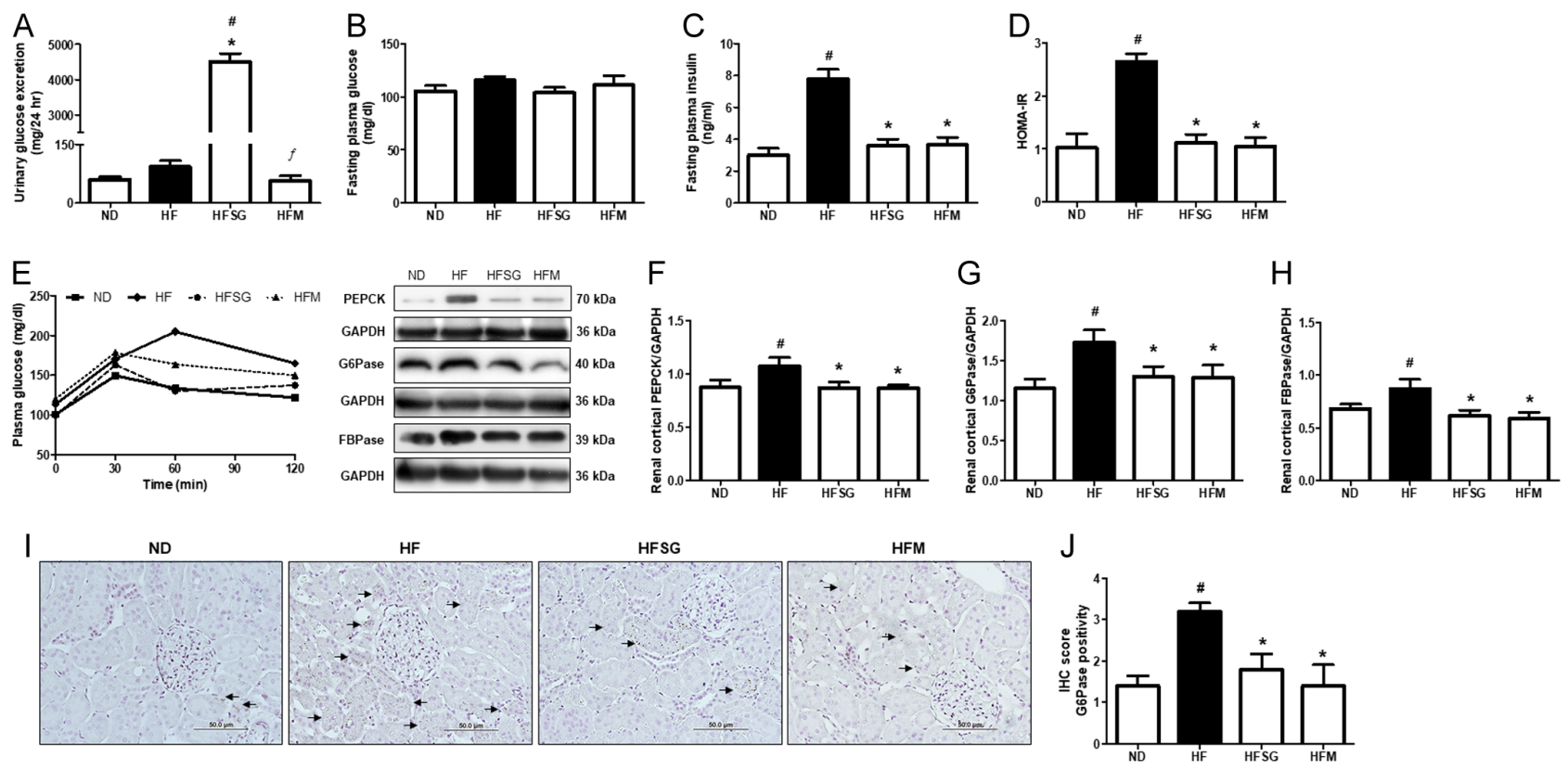

\section{Figure 1}

Effect of dapagliflozin on urinary glucose excretion, glucose metabolism and renal gluconeogenic enzyme expression in male Wistar rats. (A) Urinary glucose excretion; (B) Fasting plasma glucose level; (C) Fasting plasma insulin level; (D) HOMA-IR; (E) Plasma glucose level of OGTT and Western blot analysis of renal cortical tissue showing the expression of key gluconeogenic enzymes (F) PEPCK; (G) G6Pase; (H) FBPase in whole-cell lysate fractions; (I) Immunohistochemical staining for renal cortical G6Pase (Black arrows indicate G6Pase immunopositive cells) and (J) IHC score for renal cortical G6Pase ( $n=5$ fields/group). Results are presented as mean value for plasma glucose level of OGTT and mean \pm S.E.M. for other results. Multiple comparisons were performed by one-way ANOVA followed by Fisher's least significant difference test. $n=6$ rats/group; ND, normal diet-fed rats; HF, untreated high-fat diet-fed rats; HFSG, high-fat diet-fed rats treated with dapagliflozin; HFM, high-fat diet-fed rats treated with metformin; \# $P<0.05$ vs ND; * $P<0.05$ vs HF; $f P<0.05$ vs HFSG. A full color version of this figure is available at https://doi.org/10.1530/JOE-19-0480. 
elevations in renal gluconeogenic enzyme were concurrently reduced by not only dapagliflozin but also metformin $(P<0.05)$. The immunohistochemical staining of renal tissue also demonstrated that the increased G6Pase expression in the renal cortical tissue in $\mathrm{HF}$ rats was attenuated by dapagliflozin and metformin administration $(P<0.05)$ (Fig. 1 I and $\mathrm{J})$.

\section{The effect of dapagliflozin on renal gluconeogenic enzyme expression might be mediated through transcription factors p-GSK3 $\beta, p-C R E B$ and co-factor PGC1 1}

Forkhead box protein O1 (FOXO1), glycogen synthase kinase $3 \beta$ (GSK3 $\beta$ ), cAMP response element binding protein (CREB) and peroxisome proliferator-activated receptor-gamma coactivator 1 alpha (PGC1 $\alpha$ ) are the important transcription factors and co-factor that are reported to have significant effects on renal gluconeogenic enzyme expressions (Gatica et al. 2013, Oh et al. 2013, Wen et al. 2015, Winiarska et al. 2015, Jia et al. 2018). Unphosphorylated FOXO1, phosphoGSK3 $\beta$ (Tyr279/216) and phospho-CREB (Ser133) are the active forms of these transcription factors.
Analyzing the immunoblots from whole-cell lysate fractions of renal cortical tissue, we noted that the expression of FOXO1, GSK3 $\beta$ and CREB were not significantly changed when compared between ND and HF rats (Fig. 2A, B and D), and dapagliflozin had no effect on these protein expressions. However, metformin reduced the expression of renal cortical FOXO1 and CREB compared to $\mathrm{HF}$ rats $(P<0.05)$ (Fig. $2 \mathrm{~A}$ and $\mathrm{D})$. The expression levels of phospho-GSK3 $\beta$ ( $p$-GSK3 $\beta$ ), phospho-CREB (p-CREB) and PGC1 $\alpha$ were significantly enhanced in obese rats in comparison with ND rats $(P<0.05)$ (Fig. 2C, E and F). Dapagliflozin treatment reversed the elevated expression of these gluconeogenic transcription factors and co-factor when compared with $\mathrm{HF}$ rats $(P<0.05)$. IHC staining revealed that the number of $\mathrm{p}$-CREB positive cells in the renal cortical tissue of $\mathrm{HF}$ rats increased, while dapagliflozin treatment reduced the number $(P<0.05)$ (Fig. $2 \mathrm{G}$ and $\mathrm{H})$.

\section{Reduced renal insulin signaling in obese rats was improved by dapagliflozin}

Renal cortical insulin signaling plays a central role in regulating renal gluconeogenesis. Serine phosphorylation
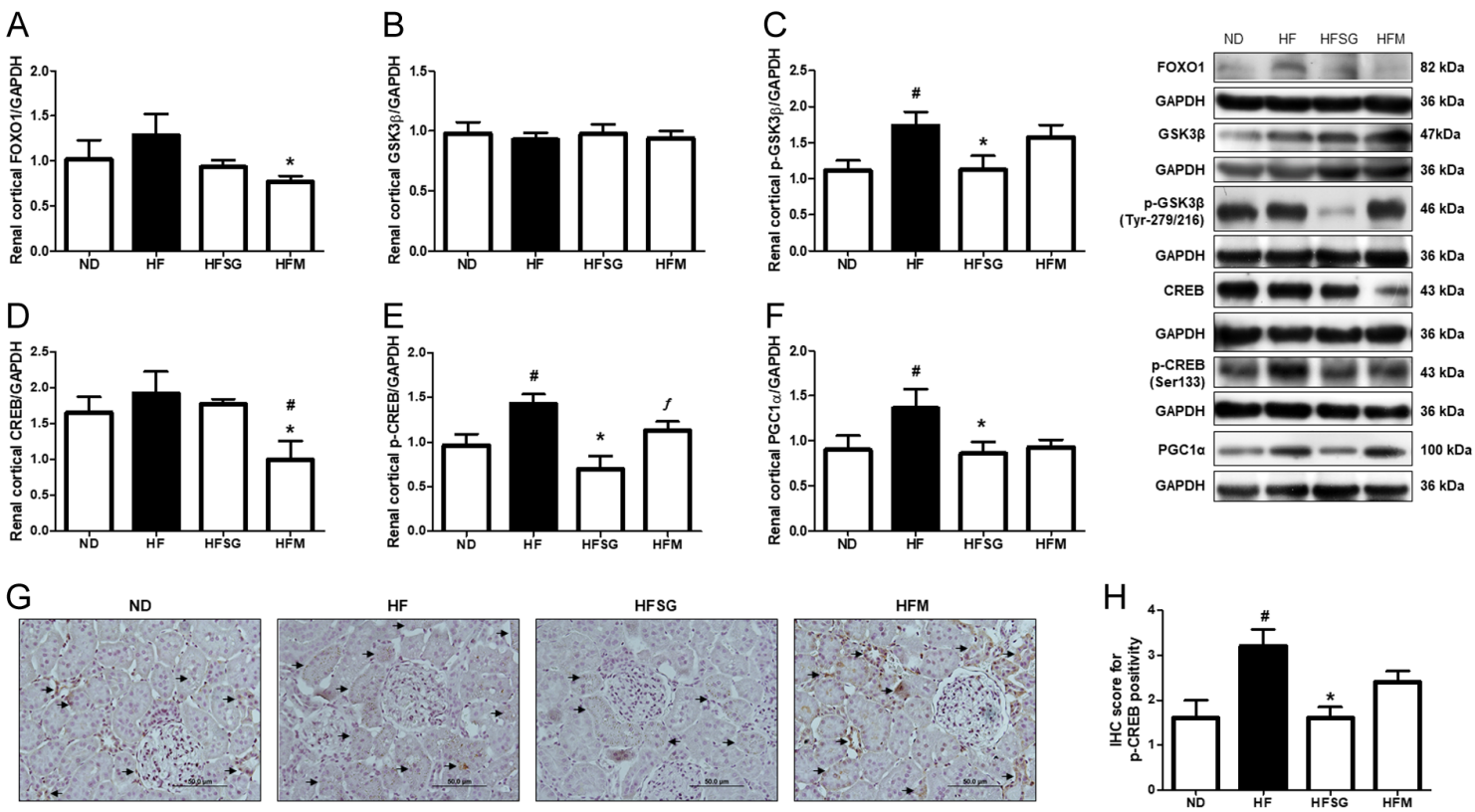

\section{Figure 2}

Effect of dapagliflozin on gluconeogenic transcription factors and co-factor in renal cortical tissue in male Wistar rats. Expression of gluconeogenic

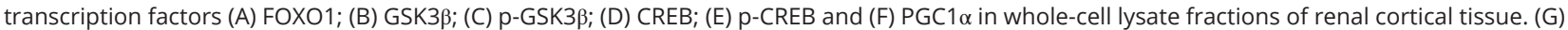
Immunohistochemical staining for renal cortical p-CREB (Black arrows indicate p-CREB immunopositive cells) and (J) IHC score for renal cortical p-CREB ( $n=5$ fields/group). Results are presented as mean \pm S.E.M. Multiple comparisons were performed by one-way ANOVA followed by Fisher's least significant difference test. $n=6$ rats/group; ND, normal diet-fed rats; HF, untreated high-fat diet-fed rats; HFSG, high-fat diet-fed rats treated with dapagliflozin; HFM, high-fat diet-fed rats treated with metformin; $\# P<0.05$ vs ND; $* P<0.05$ vs HF; $f P<0.05$ vs HFSG. A full color version of this figure is available at https://doi. org/10.1530/JOE-19-0480. 

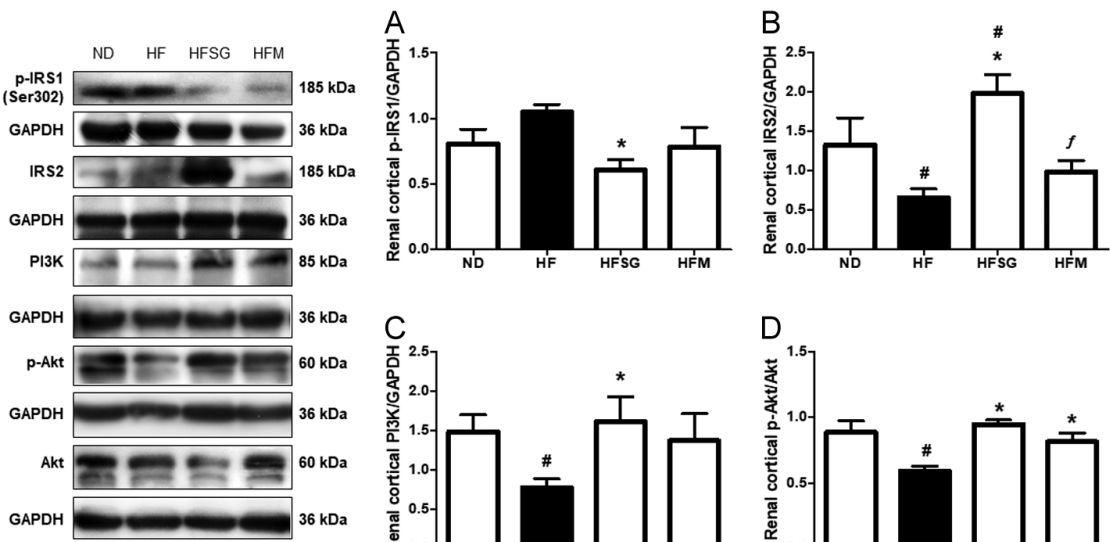

Figure 3

Effect of dapagliflozin on insulin signaling molecules in renal cortical tissue in male Wistar rats. Western blot analysis and quantification of (A) p-IRS1 (Ser302); (B) IRS2; (C) PI3K and (D) $\mathrm{p}$-Akt/Akt ratio in whole-cell lysate fractions of renal cortical tissue. Results are presented as
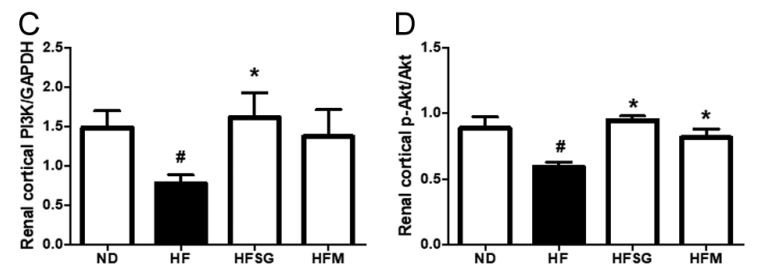
mean \pm S.E.M. Multiple comparisons were performed by one-way ANOVA followed by Fisher's least significant difference test. $n=6$ rats/group; $\mathrm{ND}$, normal diet-fed rats; HF, untreated high-fat diet-fed rats; HFSG, high-fat diet-fed rats treated with dapagliflozin; HFM, high-fat diet-fed rats treated with metformin; $\# P<0.05$ vs ND; $* P<0.05$ vs HF; $f P<0.05$ vs HFSG.

of IRS1 is regarded as the inhibitory modulation of the insulin signaling pathway (Draznin 2006). The expression of phospho-IRS1 (p-IRS1) (Serine 302) showed an increasing trend in HF rats although the increase did not reach the statistical significance $(P=0.115)$ (Fig. 3A). Dapagliflozin was found to decrease the expression level of p-IRS1 compared to the untreated group $(P<0.05)$. On the other hand, the expression levels of IRS2, PI3K and phospho-Akt (p-Akt)/Akt ratio in renal cortical tissue were significantly downregulated in $\mathrm{HF}$ rats when compared with those in ND rats $(P<0.05)$ (Fig. 3B, C and D). We found that the reduced expression of IRS2, PI3K and $\mathrm{p}$-Akt/Akt ratio in obese rats was markedly augmented by dapagliflozin $(P<0.05)$. In contrast, metformin only elevated the expression level of p-Akt/Akt ratio compared to HF rats $(P<0.05)$. Our data indicated that dapagliflozin was better than metformin in improving renal cortical post-receptor insulin signaling in obese rats.

\section{p-ERK1/2 and JNK might be the upstream mediators of the action of dapagliflozin in renal cortical tissue}

Attempting to determine the upstream regulators of transcription factors, we examined the expression level of key mediators involved in the mitogen-activated protein kinase (MAPK) pathway. Extracellular signal-regulated kinases 1/2 (ERK1/2), p38 and c-Jun N-terminal kinase (JNK) are the three signaling cassettes of the MAPK pathway, and MAPKs are redox-sensitive (Winiarska et al. 2015). In addition, JNK was reported to promote insulin resistance via serine phosphorylation of IRS molecules (Solinas and Becattini 2017). The renal cortical expression of ERK1/2, phosphoERK1/2 (p-ERK1/2) and JNK were significantly higher in the HF group than those of the ND group $(P<0.05)$ (Fig. 4A, $\mathrm{B}$ and $\mathrm{E})$. While dapagliflozin concomitantly reduced the expression of these three parameters $(P<0.05)$, metformin only decreased the expression of ERK1/2 $(P<0.05)$. On the contrary, the expression of p38 and phospho-p38 (p-p38) in renal cortical tissue did not differ among all groups (Fig. 4C and D).

5' AMP-activated protein kinase (AMPK) inhibits gluconeogenesis by inhibiting several transcription factors and also reduces NADPH oxidase 4 (NOX4)derived reactive oxygen species (ROS) level in glomerular mesangial cells (Eid et al. 2013), therefore, protecting against oxidative stress. AMPK was reported to be dysregulated in obesity (Jeon 2016). In our study, although the expression of p-AMPK was not significantly different among the experimental groups, HF rats had significantly reduced expression of AMPK, while dapagliflozin and metformin restored the reduced AMPK expression to a normal level $(P<0.05)$ (Fig. $4 \mathrm{~F}$ and $\mathrm{G})$.

\section{Renal oxidative stress in obese rats was alleviated by dapagliflozin administration}

Malondialdehyde (MDA) is a product of lipid peroxidation, which is an indicator of oxidative stress in high-fat dietfed obese rats (Noeman et al. 2011). We found that the MDA level, as assessed by the TBARS assay, was remarkably increased in the serum and renal cortical tissues of HF rats compared to ND rats $(P<0.05)$. Both were significantly reduced by dapagliflozin $(P<0.05)$ (Fig. $5 \mathrm{~A}$ and $\mathrm{B})$. In contrast, metformin only significantly attenuated renal cortical MDA level $(P<0.05)$ (Fig. 5B).

It is well known that reactive oxygen species (ROS) are usually targeted by antioxidant reduced glutathione (GSH) (Ratliff et al. 2016). In HF rats, the serum GSH level showed a decreasing trend, but the reduction was not statistically significant $(P=0.144)$ (Fig. 5C). NADPH oxidase (NOX) enzymes are the principal sources of 

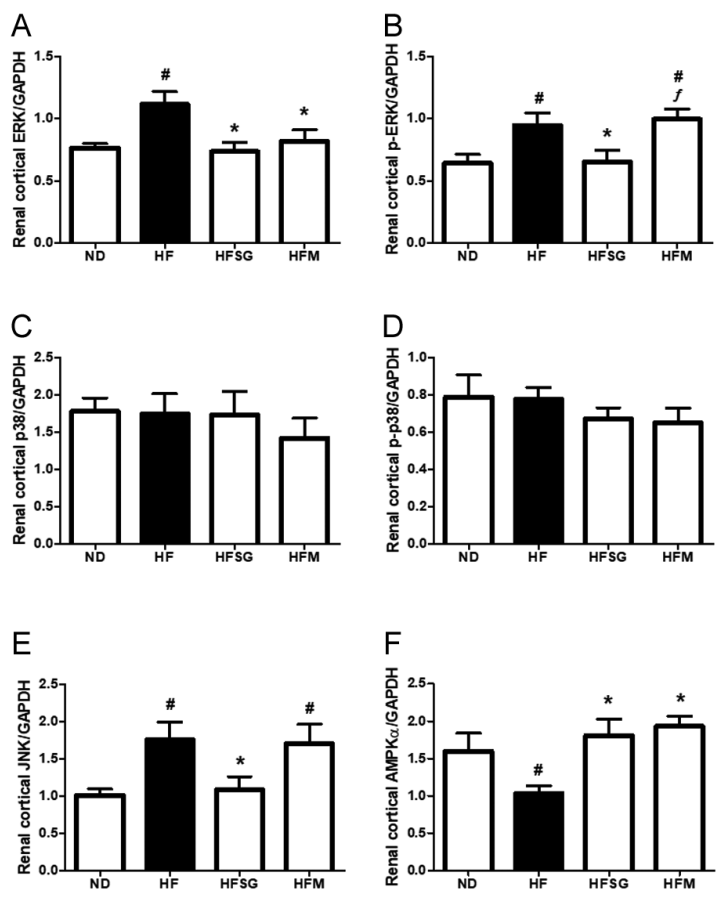

Figure 4

Effect of dapagliflozin on mediators of MAPK signaling pathway and AMPK in renal cortical tissue in male Wistar rats. Representative immunoblots and quantification of (A) ERK1/2; (B) p-ERK1/2; (C) p38; (D) p-p38; (E) JNK; (F) AMPK and (G) p-AMPK in whole-cell lysate fractions of renal cortical tissue. Results are presented as mean \pm S.E.M. Multiple comparisons were performed by one-way ANOVA followed by Fisher's least significant difference test. $n=6$ rats/group; $\mathrm{ND}$, normal diet-fed rats; $\mathrm{HF}$, untreated high-fat diet-fed rats; HFSG, high-fat diet-fed rats treated with dapagliflozin; HFM, high-fat diet-fed rats treated with metformin; $\# P<0.05$ vs ND; $* P<0.05$ vs HF; $f P<0.05$ vs HFSG.
ROS, and NOX4 isoform is highly expressed in the kidney (Ratliff et al. 2016). We found that renal cortical membrane NOX4 expression in HF rats was significantly increased which was reversed by dapagliflozin $(P<0.05)$ (Fig. 5D). These findings indicated that dapagliflozin was better than metformin in the attenuation of renal oxidative stress in obese rats.

\section{Discussion}

High-fat diet feeding for 20 weeks in male Wistar rats resulted in the increased body weight and visceral fat weight, insulin resistance, glucose intolerance and renal impairment. Dapagliflozin administration improved the metabolic profiles in obese rats and mitigated the effects of obesity on microalbuminuria, serum creatinine and creatinine clearance. The major focus of the current study was to examine the effect of dapagliflozin on renal gluconeogenic enzyme in obese rats and to elucidate the molecular signaling mechanisms underlying this effect. We demonstrated that dapagliflozin and metformin mitigated the obesity-related upregulation of renal gluconeogenic enzymes, PEPCK, G6Pase and FBPase, expression and improved glucose intolerance in obese rats. In parallel, dapagliflozin downregulated the elevated expression of gluconeogenic transcription factors p-GSK3 $\beta$ and p-CREB as well as coactivator PGC1 $\alpha$ in the renal cortical tissue. Meanwhile, metformin reduced the expression levels of transcription factors FOXO1 and CREB. In addition, the reduced renal insulin signaling was improved and the renal oxidative stress was attenuated by the treatment with dapagliflozin or metformin in obesity. Therefore, dapagliflozin improved glucose tolerance in prediabetic obese rats at least, in part, by the attenuation of the renal gluconeogenesis through improving renal cortical insulin
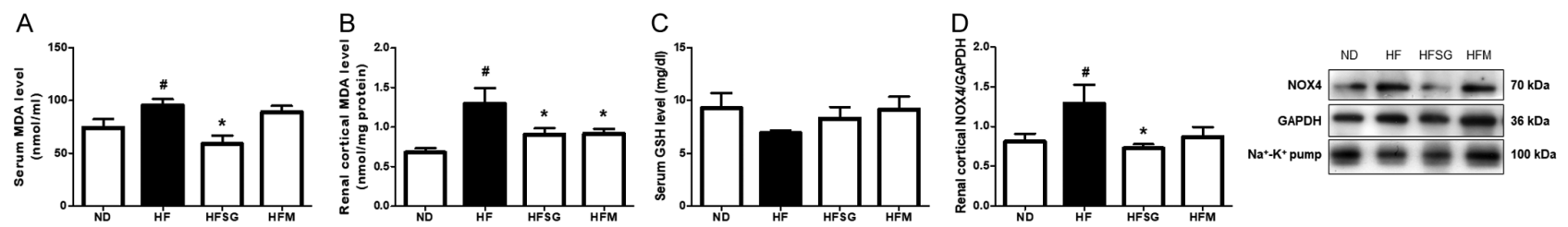

\section{Figure 5}

Effect of dapagliflozin on renal oxidative stress in male Wistar rats. (A) Serum MDA level; (B) Renal cortical MDA level; (C) Serum reduced glutathione level and (D) Western blot analysis and quantification of NOX4 in the membrane fraction of renal cortical tissue. Results are presented as mean \pm S.E.M. Multiple comparisons were performed by one-way ANOVA followed by Fisher's least significant difference test. $n=6$ rats/group; ND, normal diet-fed rats; HF, untreated high-fat diet-fed rats; HFSG, high-fat diet-fed rats treated with dapagliflozin; HFM, high-fat diet-fed rats treated with metformin; \# $P$ < 0.05 vs ND; $\star P<0.05$ vs HF; $f P<0.05$ vs HFSG. 
signaling and oxidative stress condition. Interestingly, dapagliflozin showed a higher efficacy of these effects than metformin in obese rats.

Postprandial renal glucose release is significantly increased in patients with type 2 diabetes (Meyer et al. 2004). Preclinical studies also indicated that the expression of renal gluconeogenic enzyme PEPCK was elevated in type 2 diabetic rats and concluded that renal gluconeogenesis was stimulated and crucially contributed to the hyperglycemia of type 2 diabetes (Eid et al. 2006, Wen et al. 2015, Winiarska et al. 2015). Furthermore, a study in obese rats showed that renal PEPCK expression was elevated after 24 weeks of high-fat diet feeding (Wanchai et al. 2018b). In our study, we demonstrated that the key enzymes for gluconeogenesis, PEPCK, G6Pase and FBPase, were simultaneously upregulated in the renal cortical tissue of obese, insulin resistant rats after 20 weeks of highfat diet feeding. Although the fasting plasma glucose level was not affected, these obese rats showed impaired glucose tolerance. Therefore, the elevation of renal gluconeogenic enzyme has already been established in the prediabetic obese condition which might contribute to glucose intolerance, and the involvement of renal gluconeogenesis in glucose metabolism should be emphasized not only in diabetes but also in prediabetic obesity.

However, the effects of high-fed diet-induced obesity on hepatic and renal gluconeogenic enzymes seem to be different. The activities of hepatic PEPCK and G6Pase in high-fat diet-fed rats did not significantly differ from standard chow-fed rats in a previous study (Song et al. 2001). Our previous study also found that the hepatic expression of gluconeogenic enzymes were not elevated in 20 weeks high-fat diet-fed male Wistar rats compared to normal diet-fed rats although the hepatic post-receptor insulin signaling was compromised (Swe et al. 2019b). It is possible that compensatory hyperinsulinemia was enough to suppress the hepatic gluconeogenic enzyme expression in these high-fat diet-induced obese rats.

Interestingly, the effect of SGLT2i on renal gluconeogenesis also seems to be discrepant depending on the type, dose and duration of the SGLT2i as well as the animal model, the type and severity of diabetes. Empagliflozin (300 mg/kg of regular rodent diet, approximately $60 \mathrm{mg} / \mathrm{kg} /$ day, 15 weeks) attenuated renal PEPCK mRNA expression in type 1 diabetic Akita mice (Vallon et al. 2014). An administration of dapagliflozin ( $1 \mathrm{mg} / \mathrm{kg} / \mathrm{day}, 12$ weeks) in type 2 diabetic $\mathrm{db} / \mathrm{db}$ mice resulted in further enhancement of renal gluconeogenic enzyme expression through an increase in the expression level of FOXO1 and PGC1 $\alpha$ in the kidney (Jia et al. 2018).
Contrary to this finding, empagliflozin $(10 \mathrm{mg} / \mathrm{kg} /$ day, 10 weeks) treatment in $\mathrm{db} / \mathrm{db}$ mice showed no effect on the increased mRNA expression of renal PEPCK enzyme (Gallo et al. 2016). A possible explanation is that the enhanced expression of renal gluconeogenic enzymes in diabetic condition can only be attenuated by the administration of relatively high dose of SGLT2i. It is still unclear why there is discrepancy across these studies and further research is needed to clarify this point.

In the present study, we observed that the use of dapagliflozin $(1 \mathrm{mg} / \mathrm{kg} /$ day, 4 weeks $)$ in high-fat dietfed rats attenuated the obesity-related increase in renal gluconeogenic enzyme expression. Concurrently, dapagliflozin significantly lowered glucose level during OGTT. Therefore, the administration of dapagliflozin in obesity attenuated the renal glucose release not only from renal glucose reabsorption but also possibly from renal gluconeogenesis. Thus, we proposed that attenuation of renal gluconeogenesis at least, in part, contributed to SGLT2i-mediated improvement of glucose tolerance. The use of metformin in obese rats also decreased the elevated renal gluconeogenic enzymes expression.

The renal action of dapagliflozin is on the SGLT2 cotransporter in a direct manner, and it is interesting is to observe what effect it has on other tissues. In our previous study in the same obese rat model, we found that the hepatic gluconeogenic enzymes were upregulated and hepatic and pancreatic injuries were improved by dapagliflozin treatment (Swe et al. 2019b). As the liver has no known SGLT2 expression, the hepatic action of this drug might be indirect, probably due to the metabolic changes induced by glycosuria. It is possible that an acute decrease in blood glucose due to massive urinary glucose excretion augmented the hepatic gluconeogenic program in obese rats.

Regarding the regulation of renal gluconeogenesis, insulin is the principal inhibitor of this process. The renal cortical tissues of obese rats showed post-receptor defects in insulin signaling indicated by the reduced expression of IRS2, PI3K and p-Akt/Akt ratio. Subsequently, decreased suppression of renal gluconeogenesis by insulin might lead to the elevated expression of renal gluconeogenic enzymes in untreated obese rats. Consistent with our results, a diminished renal insulin signaling was found to be associated with an upregulation of renal gluconeogenic enzymes in type 2 diabetes (Al-Quraishy et al. 2015, Wen et al. 2015). In addition, specific gene deletion of insulin receptor substrate (IRS) $1 / 2$ in the renal proximal tubule of mice resulted in impaired renal insulin signaling and elevated renal gluconeogenic gene expression (Sasaki et al. 2017). 
We observed that the reduced expressions of renal cortical IRS2, PI3K and p-Akt/Akt ratio in obese rats were restored by dapagliflozin treatment, whereas metformin improved p-Akt/Akt ratio. An improvement in postreceptor insulin signaling in renal cortical tissues after dapagliflozin treatment was found to be associated with the downregulated expression of renal gluconeogenic enzymes. In fact, insulin resistance affects not only renal gluconeogenesis but also many aspects of kidney function, including renal hemodynamics, podocyte viability and tubular function (Vallon \& Komers 2011). Consequently, dapagliflozin improved renal insulin signaling in our obese rats, resulting in the suppression of renal gluconeogenesis and amelioration of renal dysfunction.

To investigate the molecular signaling of renal gluconeogenesis, we examined the expression level of transcription factors and co-factor in the kidney cortical tissues. GSK3 $\beta$, a key kinase involved in insulin resistance and dysregulated glucose metabolism (Patel et al. 2008), positively regulates the expression of gluconeogenic enzymes in the liver (Sakamaki et al. 2012). PGC1 $\alpha$ is also one of the key positive modulators of gluconeogenesis, which is found to be elevated in the liver and kidney tissues of various models of diabetes (Yoon et al. 2001, Jia et al. 2018). The activities of GSK3 $\beta$ and PGC1 $\alpha$ are known to be inactivated by insulin signaling (Puigserver et al. 2003).

In a previous study of type 2 diabetic rats, an improvement in renal insulin signaling and subsequent
GSK3 $\beta$ inactivation was associated with the decreased expression level of renal cortical PEPCK and G6Pase protein and mRNA (Wen et al. 2015). Consistent with this study, in the renal cortical tissues of our obese rats, the attenuation of post-receptor insulin signaling was concomitant with the increases in the expression level of active p-GSK3 $\beta$, PGC1 $\alpha$ and gluconeogenic enzymes. Furthermore, an improvement in renal insulin signaling by dapagliflozin was followed by the decline in the expression of transcription factor p-GSK3 $\beta$, co-factor PGC1 $\alpha$ and renal gluconeogenic enzymes, highlighting the importance of insulin signaling and these transcription factors and co-factor in the regulation of renal gluconeogenesis in obese condition.

Insulin signaling, transduced by the insulin receptor (IR) and IRS1/2 expressed on the basolateral side of the proximal tubular cell, phosphorylates and inactivates FOXO1. An in vitro study of HK-2 cells demonstrated that FOXO1 was inactivated by insulin, accompanied by the suppression of PEPCK mRNA expression (Sasaki et al. 2017). However, in our obese rat model, the renal cortical expression of FOXO1 was not significantly higher than that of the ND rats. Dapagliflozin did not affect although metformin reduced the expression of FOXO1.

Mitogen-activated protein kinases (MAPKs) and oxidative stress conditions can alter the levels and activities of several transcription factors including gluconeogenic transcription factors (Asada etal.2007, Thornton etal.2008, Winiarska et al. 2015). Increased levels of serum and/or

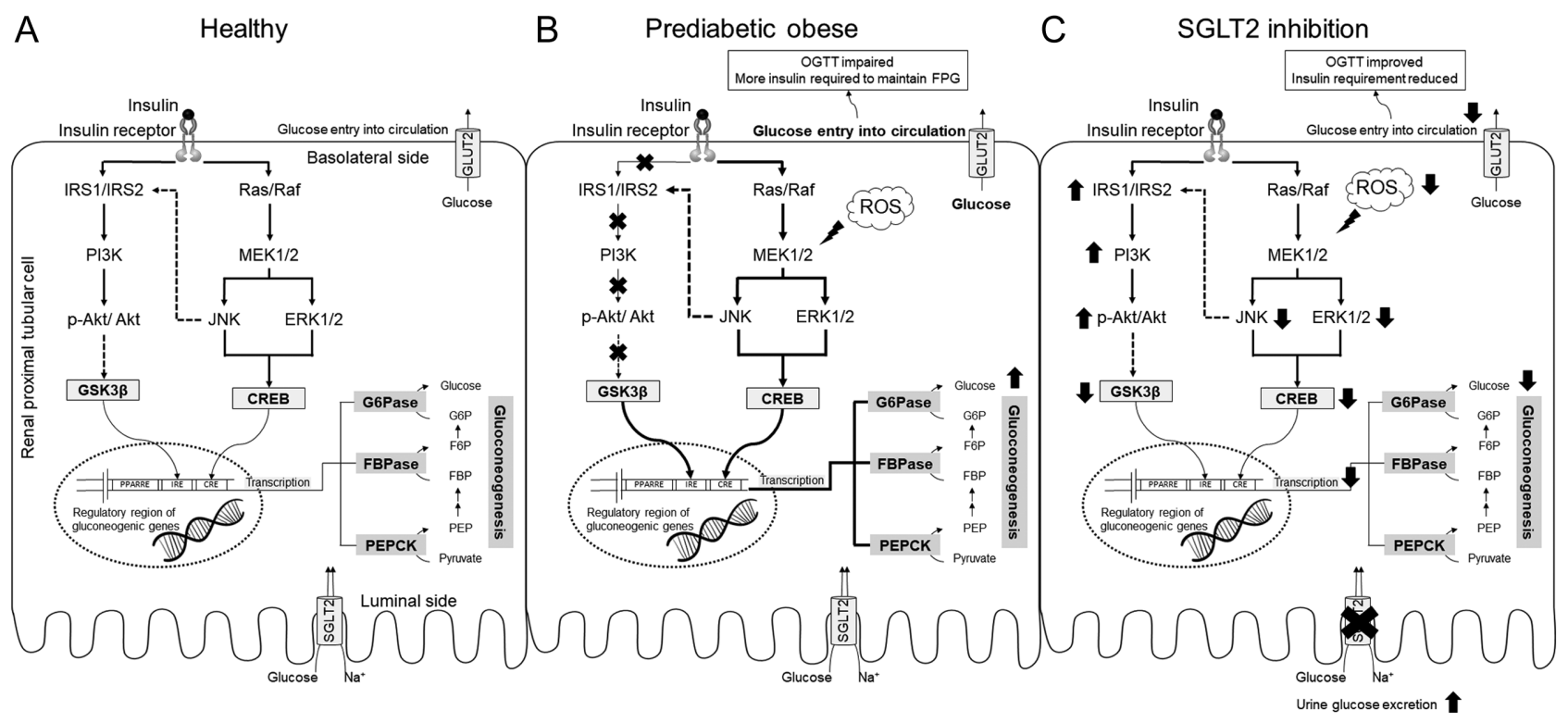

Figure 6

The effects of dapagliflozin on obesity-related overexpression of renal cortical gluconeogenic enzymes in male Wistar rats.

https://joe.bioscientifica.com https://doi.org/10.1530/JOE-19-0480 (c) 2020 Society for Endocrinology Published by Bioscientifica Ltd. Printed in Great Britain 
tissue malondialdehyde (MDA) and membrane NADPH oxidase (NOX) enzyme expression are regarded as markers of oxidative stress (Noeman et al. 2011, Ratliff et al. 2016). In addition, the NOX4 isoform of NOX enzyme plays a pivotal role in the oxidative stress related-derangement of insulin signaling in obesity (Wu \& Williams 2012).

In type 2 diabetic rats, inhibition of renal NOX enzyme led to diminished activity of PEPCK enzyme which was associated with attenuated expression of p-CREB and p-ERK1/2 in the renal cortical tissues (Winiarska et al. 2015). In our untreated obese rats, the increased levels of serum and renal cortical MDA and renal cortical membrane NOX4 expression were associated with the elevated expression of p-CREB, ERK1/2, p-ERK1/2 and JNK. Interestingly, dapagliflozin has reversed the enhanced expression of these mediators. Metformin also attenuated the renal oxidative stress, as indicated by the reduced renal cortical MDA level and ERK expression. AMPK inhibits hepatic gluconeogenesis by inhibiting various transcription factors and also protects against oxidative stress in glomerular mesangial cells (Eid et al. 2013). We found that both metformin and dapagliflozin enhanced the renal cortical expression of AMPK, although the renal p-AMPK level was not affected by either drug. Therefore, amelioration of renal cortical oxidative stress might also involve in the attenuation of obesity-induced renal gluconeogenic enzyme expression.

In summary, pharmacological inhibition of renal SGLT2 by dapagliflozin in obese rats not only reduced renal glucose reabsorption but also attenuated the obesityrelated overexpression of renal gluconeogenic enzyme expression and renal dysfunction by improving renal insulin signaling and oxidative stress. Although both dapagliflozin and metformin reduced renal gluconeogenic enzyme expression, the efficacy of dapagliflozin in improving renal insulin signaling, oxidative stress and renal function were greater than metformin. The limitation of this study is that we could only investigate the protein expression levels of renal gluconeogenic enzymes in the renal cortical tissues. It would be an advantage if we could perform in vivo measurement of renal gluconeogenesis using tracers.

\section{Conclusion}

In view of the data presented in the current study, dapagliflozin, beyond its glycosuric action, mitigated obesity-related overexpression of renal cortical gluconeogenic enzyme and renal dysfunction. The

(C) 2020 Society for Endocrinology Published by Bioscientifica Ltd. Printed in Great Britain improved insulin signaling and mitigated oxidative stress in the renal cortex might be the mechanisms underlying its effect. Interestingly, the attenuation of renal gluconeogenesis might be one of the mechanisms of SGLT2i-mediated improvement in glucose tolerance in prediabetic obesity (Fig. 6). These data suggested that the administration of dapagliflozin may be beneficial in obese prediabetic condition.

\section{Declaration of interest}

The authors declare that there is no conflict of interest that could be perceived as prejudicing the impartiality of the research reported.

\section{Funding}

This work was supported by the Thailand Research Fund (Grant number TRF-RSA 6080015 (A L)); scholarship for Doctoral degree in Physiology (International Program), Faculty of Medicine, Chiang Mai University (M S); Faculty of Medicine, Chiang Mai University (A L and A P); Graduate Research Scholarship for the Fiscal Year 2018 Graduate School Chiang Mai University (M S) and the Functional Food Research Center for Well-being, Chiang Mai University (A L).

\section{Availability of data and materials}

The datasets used and/or analyzed during the current study are available from the corresponding author on reasonable request.

\section{Author contribution statement}

$A L, A P, K J$ and $M S$ conceived and designed the experiments. A L, A P, $M S, K J$ and $L T$ conducted the experiments. $A L$ and $M S$ performed data analysis. A L, A P, M S and $L T$ performed investigation. $A L$ and $M S$ drafted the manuscript. M S performed software analysis. $A L$ and $V C$ edited and revised the manuscript. $A L, M S$ and $V C$ approved the final version of the manuscript for publication. $A \mathrm{~L}$ is the guarantor of this work and, as such, has full access to all the data in the study and takes responsibility for the integrity of the data and the accuracy of the data analysis.

\section{References}

Al-Quraishy S, Dkhil MA \& Abdel Moneim AE 2015 Anti-hyperglycemic activity of selenium nanoparticles in streptozotocin-induced diabetic rats. International Journal of Nanomedicine 10 6741-6756. (https://doi. org/10.2147/IJN.S91377)

Asada S, Daitoku H, Matsuzaki H, Saito T, Sudo T, Mukai H, Iwashita S, Kako K, Kishi T, Kasuya Y, et al. 2007 Mitogen-activated protein kinases, Erk and p38, phosphorylate and regulate FoxO1. Cellular Signalling 19 519-527. (https://doi.org/10.1016/j.cellsig.2006.08.015)

DeFronzo RA, Davidson JA \& Del Prato S 2012 The role of the kidneys in glucose homeostasis: a new path towards normalizing glycaemia. Diabetes, Obesity and Metabolism 14 5-14. (https://doi.org/10.1111/ j.1463-1326.2011.01511.x)

Draznin B 2006 Molecular mechanisms of insulin resistance: serine phosphorylation of insulin receptor substrate- 1 and increased expression of p85alpha: the two sides of a coin. Diabetes $\mathbf{5 5}$ 2392-2397. (https://doi.org/10.2337/db06-0391) 
Eid A, Bodin S, Ferrier B, Delage H, Boghossian M, Martin M, Baverel G \& Conjard A 2006 Intrinsic gluconeogenesis is enhanced in renal proximal tubules of Zucker diabetic fatty rats. Journal of the American Society of Nephrology 17 398-405. (https://doi.org/10.1681/ ASN.2005070742)

Eid AA, Lee DY, Roman LJ, Khazim K \& Gorin Y 2013 Sestrin 2 and AMPK connect hyperglycemia to Nox4-dependent endothelial nitric oxide synthase uncoupling and matrix protein expression. Molecular and Cellular Biology 33 3439-3460. (https://doi.org/10.1128/MCB.0021713)

Gallo LA, Ward MS, Fotheringham AK, Zhuang A, Borg DJ, Flemming NB, Harvie BM, Kinneally TL, Yeh SM, McCarthy DA, et al. 2016 Once daily administration of the SGLT2 inhibitor, empagliflozin, attenuates markers of renal fibrosis without improving albuminuria in diabetic db/db mice. Scientific Reports 6 26428. (https://doi.org/10.1038/ srep26428)

Gatica R, Bertinat R, Silva P, Carpio D, Ramirez MJ, Slebe JC, San Martin R, Nualart F, Campistol JM, Caelles C, et al. 2013 Altered expression and localization of insulin receptor in proximal tubule cells from human and rat diabetic kidney. Journal of Cellular Biochemistry 114 639-649. (https://doi.org/10.1002/jcb.24406)

Gerich JE 2010 Role of the kidney in normal glucose homeostasis and in the hyperglycaemia of diabetes mellitus: therapeutic implications. Diabetic Medicine 27 136-142. (https://doi.org/10.1111/j.14645491.2009.02894.x)

Gerich JE, Meyer C, Woerle HJ \& Stumvoll M 2001 Renal gluconeogenesis: its importance in human glucose homeostasis. Diabetes Care 24 382-391. (https://doi.org/10.2337/diacare.24.2.382)

Jaikumkao K, Pongchaidecha A, Chueakula N, Thongnak LO, Wanchai K, Chatsudthipong V, Chattipakorn N \& Lungkaphin A 2018 Dapagliflozin, a sodium-glucose co-transporter-2 inhibitor, slows the progression of renal complications through the suppression of renal inflammation, endoplasmic reticulum stress and apoptosis in prediabetic rats. Diabetes, Obesity and Metabolism 20 2617-2626. (https://doi.org/10.1111/dom.13441)

Jeon SM 2016 Regulation and function of AMPK in physiology and diseases. Experimental and Molecular Medicine 48 e245. (https://doi. org/10.1038/emm.2016.81)

Jia Y, He J, Wang L, Su L, Lei L, Huang W, Geng X, Zhang S, Meng X, Zhou H, et al. 2018 Dapagliflozin aggravates renal injury via promoting gluconeogenesis in $\mathrm{db} / \mathrm{db}$ mice. Cellular Physiology and Biochemistry 45 1747-1758. (https://doi.org/10.1159/000487783)

Kacerovsky M, Jones J, Schmid AI, Barosa C, Lettner A, KacerovskyBielesz G, Szendroedi J, Chmelik M, Nowotny P, Chandramouli V, et al. 2011 Postprandial and fasting hepatic glucose fluxes in long-standing type 1 diabetes. Diabetes 60 1752-1758. (https://doi. org/10.2337/db10-1001)

Khaodhiar L, Cummings S \& Apovian CM 2009 Treating diabetes and prediabetes by focusing on obesity management. Current Diabetes Reports 9 348-354. (https://doi.org/10.1007/s11892-009-0055-0)

Mather A \& Pollock C 2011 Glucose handling by the kidney. Kidney International 79 (Supplement 120) S1-S6. (https://doi.org/10.1038/ ki.2010.509)

Meyer C, Stumvoll M, Nadkarni V, Dostou J, Mitrakou A \& Gerich J 1998 Abnormal renal and hepatic glucose metabolism in type 2 diabetes mellitus. Journal of Clinical Investigation 102 619-624. (https://doi. org/10.1172/JCI2415)

Meyer C, Dostou JM, Welle SL \& Gerich JE 2002 Role of human liver, kidney, and skeletal muscle in postprandial glucose homeostasis. American Journal of Physiology: Endocrinology and Metabolism 282 E419-E427. (https://doi.org/10.1152/ajpendo.00032.2001)

Meyer C, Woerle HJ, Dostou JM, Welle SL \& Gerich JE 2004 Abnormal renal, hepatic, and muscle glucose metabolism following glucose ingestion in type 2 diabetes. American Journal of Physiology: Endocrinology and Metabolism 287 E1049-E1056. (https://doi. org/10.1152/ajpendo.00041.2004)
Mithieux G, Vidal H, Zitoun C, Bruni N, Daniele N \& Minassian C 1996 Glucose-6-phosphatase mRNA and activity are increased to the same extent in kidney and liver of diabetic rats. Diabetes 45 891-896. (https://doi.org/10.2337/diab.45.7.891)

Mitrakou A 2011 Kidney: its impact on glucose homeostasis and hormonal regulation. Diabetes Research and Clinical Practice 93 (Supplement 1) S66-S72. (https://doi.org/10.1016/S01688227(11)70016-X)

Noeman SA, Hamooda HE \& Baalash AA 2011 Biochemical study of oxidative stress markers in the liver, kidney and heart of high fat diet induced obesity in rats. Diabetology and Metabolic Syndrome 317. (https://doi.org/10.1186/1758-5996-3-17)

Oh KJ, Han HS, Kim MJ \& Koo SH 2013 CREB and FoxO1: two transcription factors for the regulation of hepatic gluconeogenesis. BMB Reports 46 567-574. (https://doi.org/10.5483/ bmbrep.2013.46.12.248)

Patel S, Doble BW, MacAulay K, Sinclair EM, Drucker DJ \& Woodgett JR 2008 Tissue-specific role of glycogen synthase kinase 3beta in glucose homeostasis and insulin action. Molecular and Cellular Biology 28 6314-6328. (https://doi.org/10.1128/MCB.00763-08)

Puigserver P, Rhee J, Donovan J, Walkey CJ, Yoon JC, Oriente F, Kitamura Y, Altomonte J, Dong H, Accili D, et al. 2003 Insulinregulated hepatic gluconeogenesis through FOXO1-PGC-1alpha interaction. Nature 423 550-555. (https://doi.org/10.1038/ nature01667)

Quinn PG \& Yeagley D 2005 Insulin regulation of PEPCK gene expression: a model for rapid and reversible modulation. Current Drug Targets: Immune, Endocrine and Metabolic Disorders 5 423-437. (https:// doi.org/10.2174/156800805774912962)

Ratliff BB, Abdulmahdi W, Pawar R \& Wolin MS 2016 Oxidant mechanisms in renal injury and disease. Antioxidants and Redox Signaling 25 119-146. (https://doi.org/10.1089/ars.2016.6665)

Rizza RA 2010 Pathogenesis of fasting and postprandial hyperglycemia in type 2 diabetes: implications for therapy. Diabetes 59 2697-2707. (https://doi.org/10.2337/db10-1032)

Roza NA, Possignolo LF, Palanch AC \& Gontijo JA 2016 Effect of longterm high-fat diet intake on peripheral insulin sensibility, blood pressure, and renal function in female rats. Food and Nutrition Research 60 28536. (https://doi.org/10.3402/fnr.v60.28536)

Sakamaki J, Daitoku H, Kaneko Y, Hagiwara A, Ueno K \& Fukamizu A 2012 GSK3beta regulates gluconeogenic gene expression through HNF4alpha and FOXO1. Journal of Receptor and Signal Transduction Research 32 96-101. (https://doi.org/10.3109/10799893.2012.660531)

Sasaki M, Sasako T, Kubota N, Sakurai Y, Takamoto I, Kubota T, Inagi R, Seki G, Goto M, Ueki K, et al. 2017 Dual regulation of gluconeogenesis by insulin and glucose in the proximal tubules of the kidney. Diabetes 66 2339-2350. (https://doi.org/10.2337/db161602)

Schulkens IA, Heusschen R, van den Boogaart V, van Suylen RJ, Dingemans AM, Griffioen AW \& Thijssen VL 2014 Galectin expression profiling identifies galectin-1 and Galectin-9Delta5 as prognostic factors in stage I/II non-small cell lung cancer. PLOS ONE 9 e107988. (https://doi.org/10.1371/journal.pone.0107988)

Shin SJ, Chung S, Kim SJ, Lee EM, Yoo YH, Kim JW, Ahn YB, Kim ES, Moon SD, Kim MJ, et al. 2016 Effect of sodium-glucose co-transporter 2 inhibitor, dapagliflozin, on renal renin-angiotensin system in an animal model of Type 2 diabetes. PLOS ONE 11 e0165703. (https:// doi.org/10.1371/journal.pone.0165703)

Solinas G \& Becattini B 2017 JNK at the crossroad of obesity, insulin resistance, and cell stress response. Molecular Metabolism 6 174-184. (https://doi.org/10.1016/j.molmet.2016.12.001)

Song S, Andrikopoulos S, Filippis C, Thorburn AW, Khan D \& Proietto J 2001 Mechanism of fat-induced hepatic gluconeogenesis: effect of metformin. American Journal of Physiology: Endocrinology and Metabolism 281 E275-E282. (https://doi.org/10.1152/ ajpendo.2001.281.2.E275) https://joe.bioscientifica.com

https://doi.org/10.1530/JOE-19-0480 (c) 2020 Society for Endocrinology Published by Bioscientifica Ltd. Printed in Great Britain 
Swe MT, Pongchaidecha A, Chatsudthipong V, Chattipakorn N \& Lungkaphin A 2019a Molecular signaling mechanisms of renal gluconeogenesis in nondiabetic and diabetic conditions. Journal of Cellular Physiology 234 8134-8151. (https://doi.org/10.1002/ jcp. 27598)

Swe MT, Thongnak L, Jaikumkao K, Pongchaidecha A, Chatsudthipong V \& Lungkaphin A 2019b Dapagliflozin not only improves hepatic injury and pancreatic endoplasmic reticulum stress, but also induces hepatic gluconeogenic enzymes expression in obese rats. Clinical Science 133 2415-2430. (https://doi.org/10.1042/CS20190863)

Terami N, Ogawa D, Tachibana H, Hatanaka T, Wada J, Nakatsuka A, Eguchi J, Horiguchi CS, Nishii N, Yamada H, et al. 2014 Longterm treatment with the sodium glucose cotransporter 2 inhibitor, dapagliflozin, ameliorates glucose homeostasis and diabetic nephropathy in db/db mice. PLOS ONE 9 e100777. (https://doi. org/10.1371/journal.pone.0100777)

Thornton TM, Pedraza-Alva G, Deng B, Wood CD, Aronshtam A, Clements JL, Sabio G, Davis RJ, Matthews DE, Doble B, et al. 2008 Phosphorylation by $38 \mathrm{MAPK}$ as an alternative pathway for GSK3beta inactivation. Science 320 667-670. (https://doi.org/10.1126/ science.1156037)

Tojo A, Hatakeyama S, Kinugasa S \& Nangaku M 2015 Angiotensin receptor blocker telmisartan suppresses renal gluconeogenesis during starvation. Diabetes, Metabolic Syndrome and Obesity: Targets and Therapy 8 103-113. (https://doi.org/10.2147/DMSO.S78771)

Vallon V \& Komers R 2011 Pathophysiology of the diabetic kidney. Comprehensive Physiology 1 1175-1232. (https://doi.org/10.1002/cphy. c100049)

Vallon V, Gerasimova M, Rose MA, Masuda T, Satriano J, Mayoux E, Koepsell H, Thomson SC \& Rieg T 2014 SGLT2 inhibitor empagliflozin reduces renal growth and albuminuria in proportion to hyperglycemia and prevents glomerular hyperfiltration in diabetic Akita mice. American Journal of Physiology: Renal Physiology 306 F194-F204. (https://doi.org/10.1152/ajprenal.00520.2013)
Wanchai K, Yasom S, Tunapong W, Chunchai T, Thiennimitr P, Chaiyasut C, Pongchaidecha A, Chatsudthipong V, Chattipakorn S, Chattipakorn N, et al. 2018 a Prebiotic prevents impaired kidney and renal Oat3 functions in obese rats. Journal of Endocrinology 237 29-42. (https://doi.org/10.1530/JOE-17-0471)

Wanchai K, Yasom S, Tunapong W, Chunchai T, Eaimworawuthikul S, Thiennimitr P, Chaiyasut C, Pongchaidecha A, Chatsudthipong V, Chattipakorn S, et al. 2018b Probiotic Lactobacillus paracasei HII01 protects rats against obese-insulin resistance-induced kidney injury and impaired renal organic anion transporter 3 function. Clinical Science 132 1545-1563. (https://doi.org/10.1042/CS20180148)

Wen Y, Lin N, Yan HT, Luo H, Chen GY, Cui JF, Shi L, Chen T, Wang T \& Tang LJ 2015 Down-regulation of renal gluconeogenesis in Type II diabetic rats following Roux-en-Y gastric bypass surgery: a potential mechanism in hypoglycemic effect. Obesity Facts 8 110-124. (https:// doi.org/10.1159/000381163)

Winiarska K, Jarzyna R, Dzik JM, Jagielski AK, Grabowski M, Nowosielska A, Focht D \& Sierakowski B 2015 ERK1/2 pathway is involved in renal gluconeogenesis inhibition under conditions of lowered NADPH oxidase activity. Free Radical Biology and Medicine $\mathbf{8 1}$ 13-21. (https://doi.org/10.1016/j.freeradbiomed.2014.12.024)

Wu X \& Williams KJ 2012 NOX4 pathway as a source of selective insulin resistance and responsiveness. Arteriosclerosis, Thrombosis, and Vascular Biology 32 1236-1245. (https://doi.org/10.1161/ ATVBAHA.111.244525)

Yoon JC, Puigserver P, Chen G, Donovan J, Wu Z, Rhee J, Adelmant G, Stafford J, Kahn CR, Granner DK, et al. 2001 Control of hepatic gluconeogenesis through the transcriptional coactivator PGC-1. Nature 413 131-138. (https://doi.org/10.1038/35093050)

Yoshihara F, Imazu M, Hamasaki T, Anzai T, Yasuda S, Ito S, Yamamoto H, Hashimura K, Yasumura Y, Mori K, et al. 2018 An exploratory study of dapagliflozin for the attenuation of albuminuria in patients with heart failure and type 2 diabetes mellitus (DAPPER). Cardiovascular Drugs and Therapy 32 183-190. (https://doi.org/10.1007/s10557-018-6782-1)

Received in final form 22 February 2020

Accepted 24 February 2020

Accepted Manuscript published online 24 February 2020 (c) 2020 Society for Endocrinology Published by Bioscientifica Ltd. Printed in Great Britain 